\title{
Effect of density and Z-contrast on the visibility of noble gas precipitates and voids with insights from Monte- Carlo simulations.
}

\author{
Anamul H Mir ${ }^{1 *}$, Jonathan. A. Hinks ${ }^{1}$, Stephen. E. Donnelly ${ }^{1}$ \\ School of Computing and Engineering, University of Huddersfield, HD1 3DH, United Kingdom
}

*Corresponding author: a.h.mir@hud.ac.uk,mirinamulhaq@gmail.com

\begin{abstract}
In this work, a detailed analysis of $\mathrm{He}, \mathrm{Ne}, \mathrm{Ar}, \mathrm{Kr}$ and $\mathrm{Xe}$ precipitates in a complex borosilicate glass using transmission electron microscopy (TEM) with in-situ ion implantation is presented. With in-situ monitoring, the real-time dynamics of precipitate and void evolution under ion implantation was followed. Using appropriate equations of state and, Monte-Carlo simulations to supplement the TEM images, we then discuss in detail the possibility and ways of differentiating the precipitates of various noble gases from empty voids. It is shown that all the noble gases precipitate as inclusions of supercritical fluid. With the aid of the simulations, the crucial role played by the size and density of the precipitates and atomic number of the gas atoms in affecting the visibility of the precipitates is highlighted. The results show that the precipitates and voids can be unambiguously differentiated in the case of $\mathrm{Xe}$ and $\mathrm{Kr}$ whereas the precipitates of other lighter noble gases cannot be differentiated from the voids. However, the precipitate and void evolution under ion irradiation follow different dynamics, knowledge of which allows one to differentiate between the precipitates and voids even for lighter noble gases. Besides shedding light on the subject of noble gas precipitation and identification of the precipitates and voids, the study highlights the complexity in dissociating the behaviour of voids from the process of precipitate re-solution. This type of knowledge is pivotal in developing models describing the evolution of precipitates, voids and macroscopic porosity in a number of materials.
\end{abstract}

Keywords: TEM, Implantation, Glass, Noble gas, Bubble, Monte-Carlo

\section{Introduction}

There is a significant interest in the behaviour and precipitation of noble gases in condensed matter. Researchers in geological and planetary sciences are interested in the estimates of noble gas solubility in minerals and as such undertake routine experiments involving noble gas infusion at high temperature and pressure. Noble gas infusion is also of great importance in fundamental glass science where the gases are used as structural probes to access the so-called "glass freevolume" which is fundamental in deciphering the structure and connectivity of the glass network. Similarly, the nuclear industry is significantly interested in understanding the behaviour and precipitation of noble gases especially $\mathrm{Xe}, \mathrm{Kr}$ and $\mathrm{He}$ in nuclear fuel (Xe and $\mathrm{Kr}$ ) structural nuclear materials ( $\mathrm{He}$ ) and, nuclear wastepackages (He) to understand the evolution and degradation, if any, of such materials due to noble gas precipitation. Furthermore, the electronics industry routinely employs noble gas implantation to tailor the dielectric and thermoelectric properties of semiconductors and insulators [13]. Due to low physical solubility and chemical reactivity (as a result of the closed shell structure), noble gases tend to precipitate at relatively low concentrations. This can result in the formation of dense solid/fluid precipitates, gaseous bubbles and voids depending on the nature of the material and temperature among some of the factors. For the sake of clarity, the terminology "bubbles" will be used to indicate the presence of a gaseous phase and "precipitate" to indicate the presence of a liquid/supercritical fluid and/or solid phase. From this standpoint, a cavity filled with noble gas atoms can be a bubble or a precipitate depending on the phase of its contents. An empty cavity, on the other hand, will be referred as a "void". In general, we will use the term "inclusions" to refer to any of these features. Amongst a plethora of techniques, transmission electron microscopy (TEM) is an indispensable tool for the study of inclusions in a variety of materials as it offers the potential to characterize and identify such precipitates at the nanoscale. The density of inclusions not only depends on the concentration of the implanted ions/infused gas but also on material properties, temperature and size of the precipitates. Clear and unambiguous identification of these inclusions is therefore important to properly understand their behaviour and dynamics. This is also crucial to develop predictive models which depend on the behaviour of precipitation, growth, size distributions etc. for nuclear materials. TEM, when combined with in-situ ion implantation, becomes a versatile instrument not only to study the inclusions themselves but also reveal the real-time dynamics taking place during the evolution of the inclusions - something that cannot be captured with any other technique at the nano-scale.

A number of TEM studies have been performed on the formation of $\mathrm{He}$ [4-13], $\mathrm{Ne}$ [13-16], $\operatorname{Ar}[12][13], \operatorname{Kr}[1,18-23]$ and $\mathrm{Xe}[1,16,18,21,24]$ precipitates in several materials. TEM diffraction studies on noble gas implanted metals (e.g. Al, Au, $\mathrm{Cu}, \mathrm{Ni})[5,7,9,16-18,20-22,24]$ have shown that dense solid precipitates of $\mathrm{Ar}, \mathrm{Kr}$ and $\mathrm{Xe}$ of few nanometres in size can be formed at room temperature. For the same implantation conditions, the size of precipitates in a given material is usually 
inversely proportional to the elastic moduli of the material [15]. Despite a number of studies on the formation of noble gas precipitates, a clear differentiation of dense precipitates, low-density gas bubbles, empty voids and the dynamics of their inter-conversion, either under ion implantation or during annealing, still remain very challenging. To date, there are no studies on the precipitates/bubbles of all the noble gases in a single material type to decipher the way in which the atom type, temperature, precipitate size and their density affect their visibility in a TEM. In fact, it is not known at all whether a distinction between the precipitates of various densities and bubbles can be made at all. Therefore, delving into this subject to clarify such issues and lay down certain basic guidelines and criteria could be helpful to a broad community dealing with noble gases and TEM.

With all these issues in mind, our goal in the current article is to present studies of the precipitates of all the noble gases (except radon) in a single material. The studies were performed on a complex borosilicate glass referred as SON68 which is a French nuclear waste glass (see Table S3 in the supplementary information for its composition). The reason to select this material for this study was its complexity which reduces the diffusion coefficient and favours the precipitation of noble gases at room temperature. The work will address some of the issues raised above and highlight the complexities and possible solutions in ambiguous situations. Furthermore, we will also present results on the simulations of the TEM images of precipitates and voids using Monte-Carlo methods to address the effect of the atom type, size and density of the precipitates on their visibility.

\section{Theoretical background}

Elastic scattering of electrons is one of the important mechanisms behind image formation in a TEM and forms the basis of the work presented in this article. The elastic scattering cross section of electrons $\left(\sigma_{\mathrm{e}}\right)$ scales as the square of the atomic number $(Z)$ of the scattering target. This is shown in equation (1) describing the relativistic Rutherford scattering:

$$
\sigma_{\mathrm{R}}(\theta)=\frac{\mathrm{Z}^{2} \lambda_{\mathrm{R}}^{4}}{64 . \pi^{4} \mathrm{a}_{0}^{2}} \frac{\mathrm{d} \Omega}{\left(\sin ^{2}\left(\frac{\theta}{2}\right)+\frac{\theta_{0}^{2}}{2}\right)^{2}}
$$

$\sigma_{R}(\theta)$ is the angle-dependent Rutherford elastic scattering cross-section; $Z$ is the atomic number of the scattering atom; $\lambda_{R}$ is the relativistic electron wavelength; $a_{0}$ is the Bohr radius of hydrogen atom $(\sim 0.1 \mathrm{~A})$; $d \Omega$ is the solid angle; $\theta$ is the scattering angle and $\theta_{0}$ is the screening parameter. Since Xe has a significantly larger $\mathrm{Z}$ than $\mathrm{He}$, it should act as a much stronger large-angle-scattering centre, deflecting a significantly higher fraction of the electrons to large angles than He. Provided a well-defined collection angle is used to collect the scattered electrons (usually by means of an objective aperture in a TEM) one can modify the intensity ratio of the scattered and nonscattered electrons to alter the contrast of the TEM micrographs. Using small collection angles will result in a large fraction of the electrons scattered from the high- $Z$ elements not being collected, which will result in a darker contrast for high-Z elements in a typical bright field (BF)-TEM micrograph. Although the strength of scattering depends on the scattering cross-section of the atoms, the amount of total scattering is also proportional to the number density of the scattering centres (density of the precipitates $(\rho)$ ). When imaging noble gas precipitates, both, $\rho$ and $\mathrm{Z}$ vary significantly and thus various noble gas precipitates lead to different contrasts depending on these two parameters.

Like any other gas, the density of noble gases strongly depends on the pressure. When implanted into materials, the internal pressure of the precipitates $(p)$ is balanced by the surface tension of the material $(\sigma)$ under equilibrium conditions. In such a scenario, the pressure and the surface tension are related through the capillary equation as given below:

$$
p=\frac{2 \sigma}{R}
$$

Where $R$ is the precipitate radius. As $\mathrm{R}$ can be directly measured from the TEM images, surface tension is the only material dependent parameter needed to evaluate the pressure. Once this is done, experimental data or a suitable equation of state (EOS) relating the pressure with the density of the noble gases is required (at a given temperature). Some of the experimental data available in the literature and the predictions of some well-known models are shown in Fig-S1 in the SI and we will be using the data presented in these graphs. The surface tension of the SON68 glass is not known, but, we consider a value of $0.25 \mathrm{~N} / \mathrm{m}$ a very reasonable estimate based on the extensive work on the surface tension of many complex glass systems [25] — with values ranging from 0.2 to $0.3 \mathrm{~N} / \mathrm{m}$, depending on the chemical composition.

\section{Materials and Methods}

3.1. Specimen preparation and ion implantation 
The glass specimens were prepared using FEI 3D quanta focussed ion beam system. Gallium ions of $30 \mathrm{keV}$ with ion current ranging from $300 \mathrm{pA}$ to $50 \mathrm{pA}$ were used to polish the lifted out specimens. Thin TEM specimens of approximately 100 $\mathrm{nm}$ thickness were obtained by a final polishing at \pm 2 degrees. All the specimens were implanted at MIAMI- 2 TEM with in-situ ion implantation facility at the University of Huddersfield, Huddersfield, United Kingdom[26]. The specimens were implanted with singly charged $\mathrm{Ne}, \mathrm{Ar}, \mathrm{Kr}$ and $\mathrm{Xe}$ ions at $295 \mathrm{~K}$. He implantations at $295 \mathrm{~K}$ did not result in any $\mathrm{He}$ precipitate formation. He implantation was then performed at $143 \mathrm{~K}$ to form the He precipitates. All the ion implantations were carried out at an angle of 18.3 degrees from the electron beam (and the specimen normal). Various implantation details are given in Table-I. The ion energy was chosen to have a similar projected ion range for all the ions, except for the He ion, which would have required significantly lower energy $(\sim 2 \mathrm{keV})$. A $300 \mathrm{keV}$ electron beam at a flux of about $6 \times 10^{17}$ electrons. $\mathrm{cm}^{-2} . \mathrm{s}^{-1}$ was used to capture the images. The electron beam was switched off during the course of ion implantation to avoid any electron beam damage and interference in the precipitate formation. Short videos requiring continuous electron beam exposure were usually recorded towards the end of the ion implantation to capture the dynamics between the precipitates and the voids.

Table-I: Singly-charged noble gas ion implantation of the SON68 glass in-situ a TEM. Implantations were performed at an angle of 18.3 degrees from the specimen surface normal (assuming the specimen to be perfectly horizontal). The ion range was calculated using SRIM software [27].

\begin{tabular}{|c|c|c|c|c|c|c|}
\hline $\begin{array}{c}\text { Ion } \\
\text { species }\end{array}$ & $\begin{array}{c}\text { Energy } \\
(\mathbf{k e V})\end{array}$ & $\begin{array}{c}\text { Flux } \\
\left(\mathbf{i o n s . c ^ { - 2 }} \mathbf{s}^{-1}\right)\end{array}$ & $\begin{array}{c}\text { Irradiation } \\
\text { Temperature } \\
(\mathbf{K})\end{array}$ & $\begin{array}{c}\text { Maximum } \\
\text { irradiation } \\
\text { fluence } \\
\text { (ions.cm }\end{array}$ & $\begin{array}{c}\text { Projected } \\
\text { range } \\
(\mathbf{n m})\end{array}$ & $\begin{array}{c}\text { Formation } \\
\text { of } \\
\text { precipitates } \\
\text { and voids }\end{array}$ \\
\hline${ }^{4} \mathrm{He}^{+}$ & 6 & $1.1 \times 10^{13}$ & 295 & $5 \times 10^{16}$ & $55 \pm 25$ & No \\
\hline${ }^{4} \mathrm{He}^{+}$ & 6 & $1.2 \times 10^{13}$ & 143 & $1 \times 10^{17}$ & $55 \pm 25$ & Yes \\
\hline${ }^{20} \mathrm{Ne}^{+}$ & 15 & $2.5 \times 10^{13}$ & 295 & $5.1 \times 10^{16}$ & $27 \pm 12$ & Yes \\
\hline${ }^{40} \mathrm{Ar}^{+}$ & 18 & $2.8 \times 10^{13}$ & 295 & $1.7 \times 10^{16}$ & $19 \pm 7$ & Yes \\
\hline${ }^{84} \mathrm{Kr}^{+}$ & 35 & $1.6 \times 10^{13}$ & 295 & $2.8 \times 10^{16}$ & $22 \pm 7$ & Yes \\
\hline${ }^{84} \mathrm{Kr}^{+}$ & 30 & $2.2 \times 10^{13}$ & 295 & $1.2 \times 10^{16}$ & $20 \pm 6$ & Yes \\
\hline${ }^{84} \mathrm{Xe}^{+}$ & 45 & $1.2 \times 10^{13}$ & 295 & $3.1 \times 10^{16}$ & $24 \pm 6$ & Yes \\
\hline${ }^{84} \mathrm{Xe}^{+}$ & 45 & $2.1 \times 10^{13}$ & 295 & $1.9 \times 10^{16}$ & $24 \pm 6$ & Yes \\
\hline
\end{tabular}

\subsection{Monte-Carlo Simulations}

The Monte-Carlo simulations were carried out to simulate the scattering of electrons and reproduce the 2D-TEM images using the scattered electrons. The simulations were performed with the freely available Casino software [28-31] The simulations were performed with an electron beam energy of $300 \mathrm{keV}$ using 10000 electrons per spot. A beam spot size of $0.6 \mathrm{~nm}$ and a distance of $0.3 \mathrm{~nm}$ between the spots was used to generate the two-dimensional simulated TEM images. All the simulations used ELSEPA (elastic scattering of electrons and positrons) elastic scattering cross sections [32].

\section{Results}

BF-TEM images of the SON68 glass implanted with various noble gases are shown in Fig.1 (rows R1-R5 for Xe to He respectively). The images were captured using a collection angle of $9 \mathrm{mrad}$ for $\mathrm{He}$ and $34 \mathrm{mrad}$ for the other gases (by using different objective apertures). The images are shown after fluences of $1.9 \times 10^{16}, 1.9 \times 10^{16}, 6.4 \times 10^{16}, 5.4 \times 10^{16}$ and $7 \times 10^{16}$ ions.cm ${ }^{-2}$ in rows R1 to R5 for ${ }^{132} \mathrm{Xe},{ }^{84} \mathrm{Kr},{ }^{40} \mathrm{Ar},{ }^{20} \mathrm{Ne}$ and ${ }^{4} \mathrm{He}$ respectively. As a general example, the evolution of the precipitate sizes as a function of the implantation fluence is shown in Fig. S2 in the SI for the case of Xe. Figure 1 shows under-focus, at-focus and over-focus images on the left-hand side, in the middle and on the right-hand side respectively in all the rows from R1 to R5. The voids and the precipitates are indicated by the white (solid) and yellow (dash) arrows in Fig. 1 (R1-R3) respectively. The dark precipitates and the bright voids are distinctly visible in the case of Xe in the overfocus and at-focus images. The precipitates are less distinctly visible in the under-focus images. The intensity profiles on the extreme right taken along the lines indicated on the figures represent the transmitted electron intensities (single pixel line scans). The ratio of the intensity transmitted through a void $\left(\mathrm{I}_{\mathrm{void}}\right)$ and a precipitate $\left(\mathrm{I}_{\mathrm{prp}}\right)$ written underneath each line profile is about $1.15 \pm 0.3$ for $\mathrm{Xe}$. In going from $\mathrm{Xe}$ to $\mathrm{Kr}$, the precipitates appear lighter relative to the Xe precipitates, but, there is still a clear difference between the precipitates and the voids under all the focusing conditions. The void to precipitate intensity ratio is about $1.07 \pm 0.5$ for $\mathrm{Kr}$. This corresponds to about $7 \%$ drop in comparison to Xe. In the case of Ar, it is very difficult to have an easy and unambiguous distinction between the voids and the precipitates. There is 
essentially no change in the void to precipitate intensity ratio when compared to $\mathrm{Kr}$. The possible precipitates and voids are indicated by the arrows as earlier. A more detailed discussion on the subject of Ar precipitates will follow later. For the case of $\mathrm{Ne}$ and $\mathrm{He}$, there is no distinction between the voids and the precipitates. A few irregular dark features seen in the case of Ne are surface contamination that was present from the beginning of the experiment. Some bright spots seen in the case of $\mathrm{He}$ (as indicated by the red arrows) are due to an overlap between the precipitates/voids at different depths; yielding a higher transmitted intensity. The overlap is more clearly evident from the over-focus images in which overlapping Fresnel fringes can be seen with higher brightness in the union of the two fringes (see Fig-S3 in the SI for higher magnification images).

Amongst the implanted noble gases, the precipitate overlap was significantly and prominently seen in the case of He only. The reason behind this overlap is the fact that He implantation profile is very broad as compared to other noble gases. The implantation profiles become broader with a decrease in the $\mathrm{Z}$ of the implanted atom (see Fig S3 in the SI) due to a higher degree of straggling for lighter atoms. A broader implantation profile (and deeper) results in He being distributed over a larger depth. Consequently, the critical concentration needed to form the precipitates is satisfied over a wide range as the implantation continues to higher fluences. This results in the growth of large He precipitates along the implanted depth whose images overlap -in some cases where they are in close proximity, bubbles and precipitates may physically merge into a bigger one. This is usually seen during the annealing as evident from Fig. S4 in the SI. Thus, when comparing the precipitates of various noble gases, there is a significant difference in terms of their contrast and appearance - which besides density, strongly depend on the $\mathrm{Z}$ of the implanted gas atoms. Heavier atoms form relatively darker precipitates whereas light atoms form relatively bright precipitates on the glass background. The glass background is mainly a result of scattering from low-Z elements such as oxygen, silicon, boron and sodium constituting the glass (which together make about 90 atomic $\%$ of the SON68 glass). 

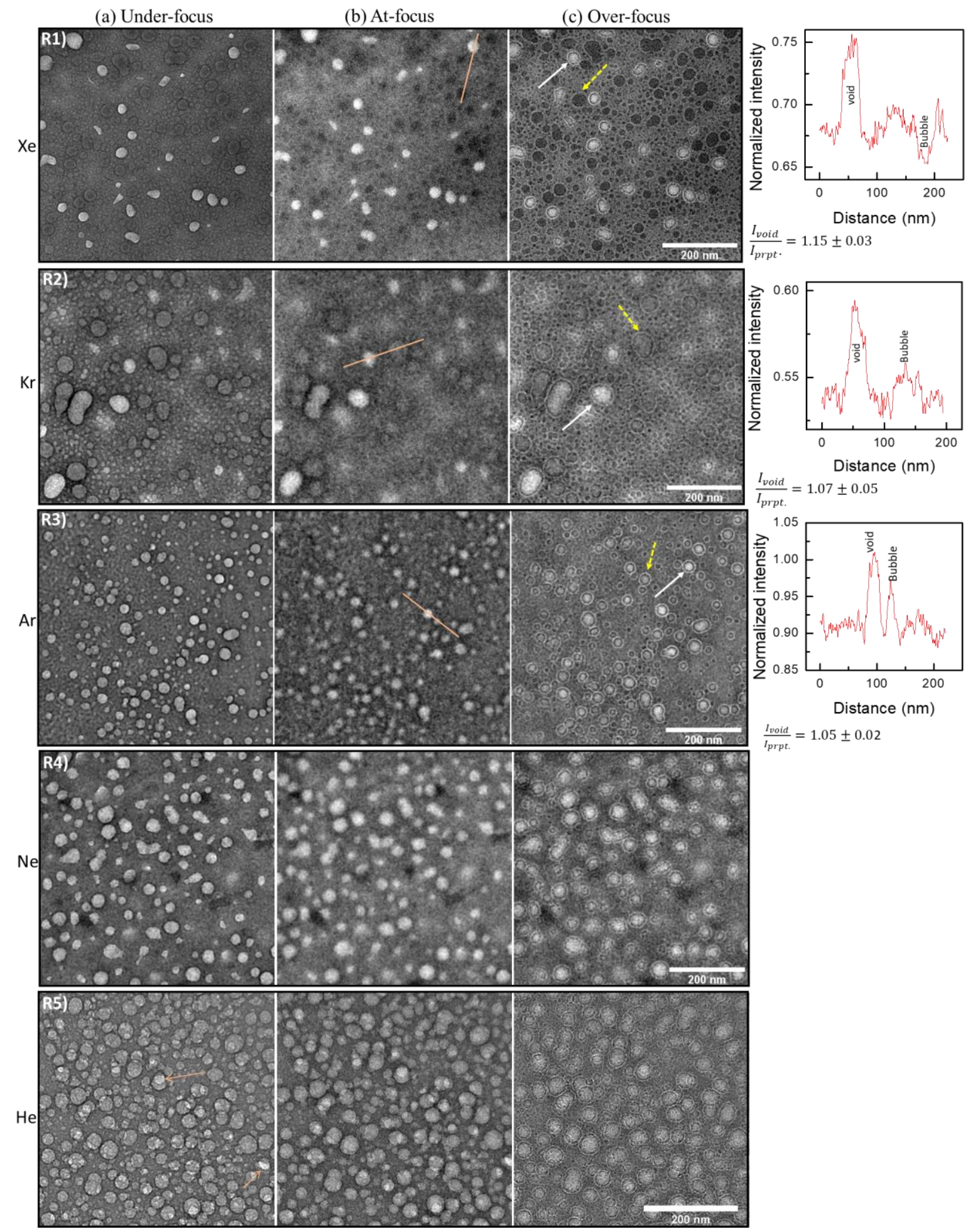

Figure-1:BF-TEM images showing noble gas precipitates in the SON68 glass after in-situ implantation. (R1-R5) underfocus, at-focus, and over-focus images (from a-c respectively) after implantation with Xe (R1), $\mathrm{Kr}(R 2), \mathrm{Ar}(R 3), \mathrm{Ne}(R 4)$ and $\mathrm{He}(\mathrm{R} 5)$ up to fluences of $1.9 \times 10^{16}(R 1) 1.9 \times 10^{16}(R 2) 6.4 \times 10^{16}(R 3) 5.4 \times 10^{16}(R 4)$, $7 \times 10^{16}(R 5)$ ions.cm ${ }^{-2}$ respectively. The profiles in the red colour show normalized transmitted intensity line scans across the lines shown on the at-focus images. All the ions except He were implanted at $295 \mathrm{~K}$. He was implanted at $143 \mathrm{~K}$ (defocus $= \pm 4 \mu \mathrm{m}$, scale markers apply to all the images in a given row).

\section{Discussion}

\subsection{Typical pressure, density and physical state of the noble gas precipitates}

Before further discussing the BF-TEM micrographs shown in Fig. 1, it is worth estimating the typical density of the precipitates seen in the TEM micrographs. This can be estimated from the P-V data and EOS predictions shown in Fig. S1 in the SI. 
The typical lower and upper limits of the precipitate diameters seen during the implantation of various noble gases are listed in Table-II (most of the precipitates seen in the TEM micrographs in Fig. 1 are closer to the upper limit). Based on the limits of the diameter, the lower and upper limits of the precipitate pressure calculated using equation (2) are also listed in TableII. The lower and upper-density limits shown in Table-II were then obtained from the data in Fig. S1 in the SI. This shows that the densest $\mathrm{He}, \mathrm{Ne}, \mathrm{Ar}, \mathrm{Kr}$ and Xe precipitates contain less than 0.2, 0.8, 1.2, 2.3 and $3{\mathrm{~g} . \mathrm{cm}^{-3}}^{-3}$ of the respective noble

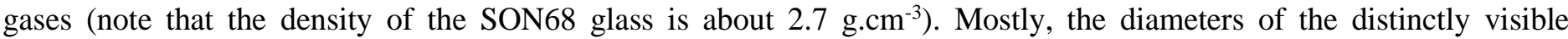
precipitates in Fig. 1 are closer to the upper limit of the diameter listed in Table-II. Therefore, their densities are closer to the lower limit of the density listed in Table-II. Also, based on the melting curves of various noble gases shown in Fig. S1 in the SI, the pressures needed for the solid precipitates to exist at room temperature $(300 \mathrm{~K})$ are listed in Table-II. For the solid phase to exist at room temperature, high-pressures ranging from $440 \mathrm{MPa}$ to $11.7 \mathrm{GPa}$ are needed for Xe and $\mathrm{He}$ respectively. The other gases require pressures between these two limits.

To highlight the size dependence of the pressure and density of the precipitates, the data for $\mathrm{Kr}$ and $\mathrm{Xe}$ precipitates as a function of their diameter is shown in Fig. S1 in the SI. The precipitate diameters that can sustain pressures for the solid precipitates to exist are listed in Table-II for all the gases. For the case of $\mathrm{He}$ and $\mathrm{Ne}$, solid state precipitates are of subnanometre size whereas for the other gases relatively large solid precipitates can exist at $300 \mathrm{~K}$. The critical constants of the noble gases are shown in Table S1 in the SI. The critical temperatures of all the noble gases lie below the implantation temperature used for the respective gases. Also, the critical pressures listed in Table-S1 in the SI show that even the largest visible precipitates in Fig. 1 have pressures far exceeding the critical pressure of the respective noble gases. Therefore, considering the critical constants of the noble gases and the pressure limits given in Table-II, it is clear that all the noble gas precipitates exist as supercritical fluid inclusions at their respective implantation temperatures. From equation (1), it is clear that high surface tension and very small precipitates will facilitate the formation of a solid phase. Generally, metals have high surface tensions which facilitates the formation of relatively large solid precipitates even at room temperature (for instance, with a surface tension of about $2.5 \mathrm{~N} / \mathrm{m}$, tungsten can sustain up to $20 \mathrm{~nm}$ large solid precipitates of Xe). For the glasses, the surface tensions are much lower, and only very small precipitates can exist in the solid phase at room temperature (as listed in Table-II). The TEM diffractograms of the specimens implanted with various noble gases confirmed the lack of any measurable crystalline inclusions at their respective implantation temperatures. Thus, going forward, it is important to bear in mind that the noble gas precipitates in glasses exist as inclusions of the supercritical fluid, which would require a depressurization of few hundred MPas to transform into a distinct gas phase to be referred as gas bubbles.

Table-II: Typical lower and upper diameters $\left(D_{l}, D_{u}\right)$, lower and upper limit of pressures $\left(P_{l}, P_{u}\right)$ and lower and upper limit of densities $\left(\rho_{l}, \rho_{u}\right)$ for the precipitates seen in the TEM micrographs. The pressures were calculated using equation (2) and the densities using the data in Fig. S1 in the SI. The last two columns show the pressure and precipitate diameters required to have a solid phase at $300 \mathrm{~K}$.

\begin{tabular}{|c|c|c|c|c|c|c|c|c|}
\hline $\begin{array}{c}\text { Implanted } \\
\text { ion }\end{array}$ & $\begin{array}{c}\mathbf{D}_{\mathbf{l}} \\
(\mathbf{n m})\end{array}$ & $\begin{array}{c}\mathbf{D}_{\mathbf{u}} \\
(\mathbf{n m})\end{array}$ & $\begin{array}{c}\mathbf{P}_{\mathbf{1}} \\
(\mathbf{M P a})\end{array}$ & $\begin{array}{c}\mathbf{P u}_{\mathbf{u}} \\
(\mathbf{M p a})\end{array}$ & $\begin{array}{c}\mathbf{\rho} \mathbf{l} \\
(\mathbf{g} / \mathbf{c c})\end{array}$ & $\begin{array}{c}\mathbf{\rho u} \\
(\mathbf{g} / \mathbf{c c})\end{array}$ & $\begin{array}{c}\text { Solidification } \\
\mathbf{p r e s s u r e} \text { at 300 } \\
\mathbf{K}(\mathbf{G P a})\end{array}$ & $\begin{array}{c}\text { Precipitate } \\
\mathbf{d i a m e t e r} \\
\text { required for the } \\
\mathbf{s o l i d ~ s t a t e ~ t o ~ e x i s t ~} \\
(\mathbf{n m})\end{array}$ \\
\hline $\mathbf{H e}$ & 5 & 40 & 25 & 200 & 0.03 & 0.18 & 11.7 & 0.08 \\
\hline $\mathbf{N e}$ & 5 & 35 & 29 & 200 & 0.2 & 0.8 & 4.8 & 0.2 \\
\hline $\mathbf{A r}$ & 5 & 30 & 33 & 200 & 0.5 & 1.2 & 1.35 & 0.8 \\
\hline $\mathbf{K r}$ & 5 & 40 & 25 & 200 & 1 & 2.3 & 0.84 & 1.2 \\
\hline $\mathbf{X e}$ & 5 & 30 & 33 & 200 & 2.3 & 3 & 0.44 & 2.2 \\
\hline
\end{tabular}

\subsection{Insights from Monte-Carlo (MC) simulations}

\subsubsection{Effect of specimen thickness and collection angle}

The MC simulations were performed under similar conditions as used for acquiring the TEM images (electron energy, collection angle, specimen thickness etc). It is important to bear in mind that these simulations do not consider the wave nature of the electrons and therefore the out of focus images — which have a dominant phase contribution as well—can not be simulated. The simulations are valid for at-focus images only, where $\mathrm{Z}$ and density contrast are the dominant factors. As a representative case, the case of He precipitates will be described. A total of 11 spherical inclusions of $10 \mathrm{~nm}$ diameter were embedded inside the SON68 glass matrix. The spherical inclusions were then filled with He atoms with density ranging from 0 (void) to $5 \mathrm{~g} . \mathrm{cm}^{-3}$ in increments of $0.5 \mathrm{~g} . \mathrm{cm}^{-3}$ between the inclusions. The actual thickness of the SON68 specimens 
is not precisely known, but, the relative specimen thickness estimated from the EELS spectra varied from 0.5 to 1.4 mean free paths between different specimens. Assuming that the inelastic mean free path of $300 \mathrm{keV}$ electrons in the SON68 glass is similar to that in a-SiO 2 and modified only due to the higher density of the $\mathrm{SON}_{6}$ glass relative to a-SiO${ }_{2}\left(\mathrm{SON}_{6} 8\right.$ is $22 \%$ denser than $\mathrm{a}-\mathrm{SiO}_{2}$ ), then the thickness of the specimens can be expected to be between 80 to $300 \mathrm{~nm}$ based on the inelastic mean free path value of a- $\mathrm{SiO}_{2}$ [33] (the mean free path is directly proportional to the molecular weight and inversely proportional to the product of density and inelastic scattering cross-section [34]. The molecular weight of $\mathrm{SiO}_{2}$ and SON68 is about 60 grams per mole; therefore, the density plays the dominant role because the major atomic species in both the glasses are similar).

The simulated images and transmitted electron intensity profiles showing the effect of specimen thickness ' $t$ ' (100, 200 and $300 \mathrm{~nm}$ ) and electron collection angle ' $\theta_{\mathrm{c}}$ ' (defined by the objective aperture in a TEM) are shown in Fig. S5 in the SI. A simple visual inspection of the images revealed that the brightness decreased with an increase in the specimen thickness. This is intuitive and consistent with the Beer-Lambert law [35]. Only the overall brightness of various features was affected by ' $t$ ' and ' $\theta_{c}$ ', but, none of these two parameters had a dominant effect on the visibility of various inclusions and their differentiation from each other (a detailed discussion on the quantitative values of transmitted intensity, contrasts etc for different specimen thicknesses and collection angles are given in the SI). The transmitted intensity decreased with an increase in the specimen thickness and a decrease in the collection angle. From the results, we conclude that the collection angle does not play as significant a role in thin specimens as in thick specimens in affecting the transmitted intensity. This behaviour is because the collision probability (single and multiple) increases with an increase in the specimen thickness. Consequently, more electrons are removed from the primary beam and scattered to higher angles as the specimen thickness increases. The collection angle then becomes important in controlling the fraction of the scattered electrons that are within ' $\theta_{c}$ '. For thin specimens, most of the electrons pass without any large angle scattering and collection angle plays a less significant role. Furthermore, smaller collection angle gave a better image contrast. Thus, other than changing the global brightness levels, specimen thickness in the range of 100 to $300 \mathrm{~nm}$ and collection angles in the range of 9 to $34 \mathrm{mrad}$ did not have a significant bearing on the visibility and differentiation of He precipitates and voids. For heavier elements, a small but measurable impact of specimen thickness on the visibility of the precipitates was observed which will be discussed later.

\subsubsection{Effect of precipitate density on their visibility}

Based on the discussion above, we will only focus on a specimen of $100 \mathrm{~nm}$ thickness assuming that the thickness does not play a major role (any small role that it plays for heavier elements will be pointed out later). As before, 11 precipitates of

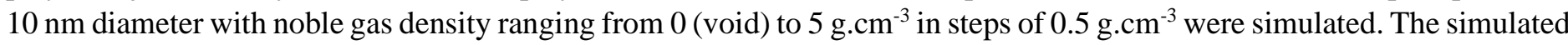
BF-TEM micrographs for various noble gas precipitates are shown in Fig. 2 (R1, R5) for He, Ne, Ar, Kr and Xe respectively. For $\mathrm{He}$, the simulation results are shown for a collection angle of $9 \mathrm{mrad}$. For the rest of the noble gases, the results are shown for a collection angle of $34 \mathrm{mrad}$. At-focus experimental TEM micrographs of $\mathrm{Ar}, \mathrm{Kr}$ and $\mathrm{Xe}$ are also shown on the right-hand side of the simulated images. The densities of various precipitates are written underneath image R5. The horizontal colour bars indicate the typical density range of the precipitates seen in the experimental TEM micrographs and listed in Table-I (upper and lower limits). The yellow circles indicate the actual equilibrium density of a $10 \mathrm{~nm}$ precipitate. Therefore, the precipitates on the left-hand side of the orange circle are over-pressurized and, are used in our simulation only to highlight the effect of the precipitate density on their visibility. The images in Fig. 2 essentially show the effect of ' $Z$ ' along the vertical direction and the effect of ' $\rho$ ' along the horizontal direction on the visibility of precipitates. The simulated transmitted intensity line scans going through the centre of the precipitates from left to right-hand side are shown in Fig. 2(a). Irrespective of the He density in the studied range, He precipitates appear as bright features. From the intensity line scans in Fig. 2 (a), it is clear that a void cannot be distinguished from a He precipitate in the density range of our interest $\left(0.03\right.$ to 0.18 g.cm $\left.{ }^{-3}\right)$.

For He precipitates in other materials with surface tension an order of magnitude higher than glasses (for instance, tungsten has a surface tension of about $2.5 \mathrm{~N} / \mathrm{m}$ ) and He precipitate sizes of about $1 \mathrm{~nm}$ diameter (which are highly pressurized and dense), the equilibrium pressure and density would be about $5.4 \mathrm{GPa}$ and $0.6 \mathrm{~g} . \mathrm{cm}^{-3}$, respectively. The simulation results presented above show that even at these high He precipitate densities, it will not be possible to visually distinguish between a He precipitate and a void in TEM micrographs. Helium precipitate densities greater than $1 \mathrm{~g} . \mathrm{cm}^{-3}$ are unrealistically high for the precipitates generally formed during ion implantation. Therefore, a clear distinction between voids and $\mathrm{He}$ precipitates formed during ion implantation of glasses and even of materials with much higher surface tension is simply not 
possible without the use of an analytical technique. These results are consistent with the experimental TEM micrographs of He precipitates in Fig. 1 (R5), which did not show any visible brightness variations among the observed precipitates/voids.

For the case of Xe precipitates shown in Fig. 2 (R5), the simulations predicted the appearance of dark features in the entire density range of our interest ( 2 to $3 \mathrm{~g} \mathrm{~cm}^{-3}$ ). This is in excellent agreement with the experimental at-focus image of Xe precipitates shown on the right-hand side (it is a small cropped region from Fig. 1 (R1)). The precipitates became darker as the density was further increased. Precipitates with density less than $1 \mathrm{~g} . \mathrm{cm}^{-3}$ appeared as bright features. However, even a precipitate of just $1 \mathrm{~g} . \mathrm{cm}^{-3}$ can be visually distinguished from a void in the simulated TEM micrographs. Precipitates in the density range of $1<\rho<2 \mathrm{~g} . \mathrm{cm}^{-3}$ were invisible. In order to confirm that the precipitates in a specific density range should be invisible, high-temperature annealing experiments to slowly decrease the density of Xe precipitates through diffusion mediated Xe losses were performed. These experiments clearly confirmed these predictions showing a transition from dark appearing precipitates to invisible and, eventually to bright features for low-density precipitates and voids (see Fig. 4 in [36] for more details). When comparing the results of $100 \mathrm{~nm}$ and $200 \mathrm{~nm}$ thick specimens, precipitates of 2 g.cm ${ }^{-3}$ were visible in the $100 \mathrm{~nm}$ thick specimens, but, completely invisible in the $200 \mathrm{~nm}$ thick specimen (see Fig. S6 in the SI). Thus, unlike He precipitates, specimen thickness affected the visibility of Xe precipitates. This highlighted that the specimen thickness and precipitate density were strongly convoluted.
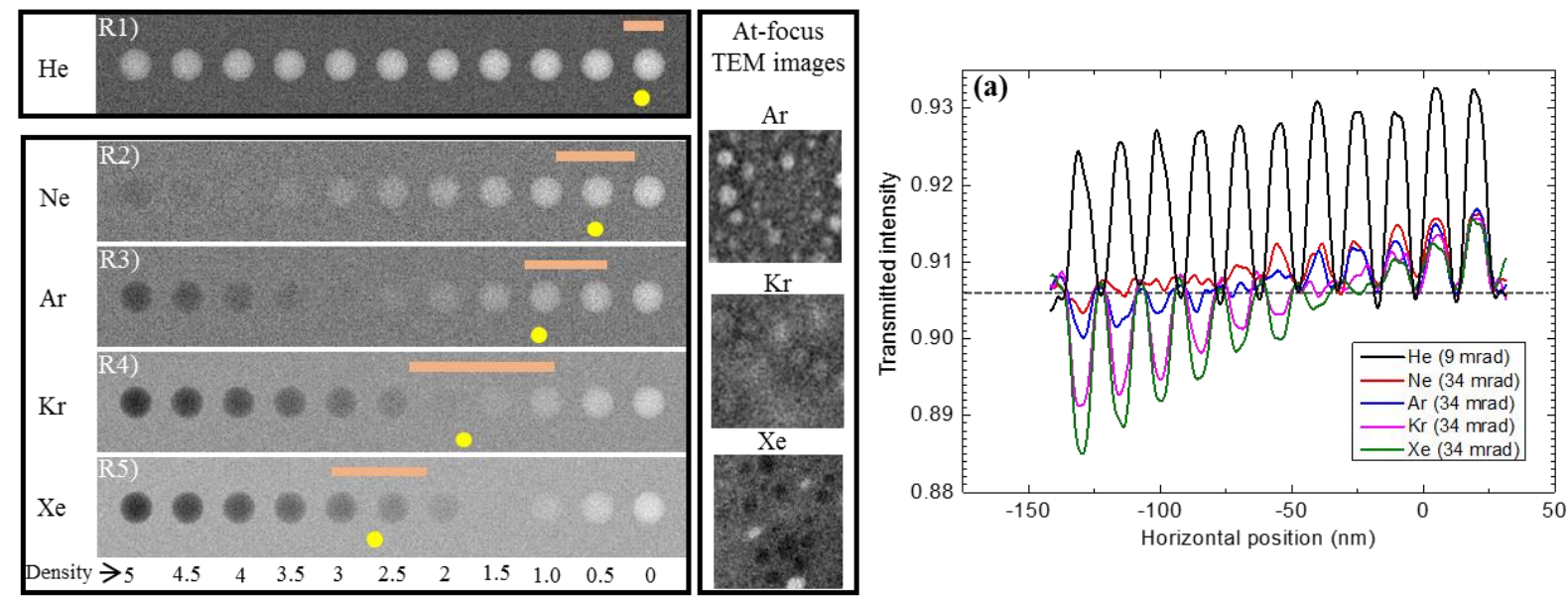

Figure-2: Monte-Carlo simulation of noble gas precipitates in the SON68 glass. (Rows R1-R5), simulated BF-TEM micrographs of $10 \mathrm{~nm}$ diameter precipitates of $\mathrm{He}, \mathrm{Ne}, \mathrm{Ar}, \mathrm{Kr}$ and Xe in a $100 \mathrm{~nm}$ thick SON68 glass specimen. The density

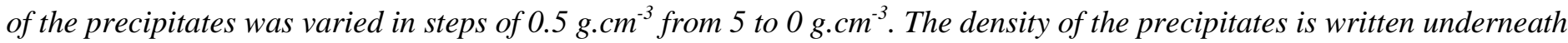
image R5. The orange colour bars show the density range of the precipitates seen in the experimental TEM micrographs and listed in Table-II. The circles in orange indicate the equilibrium density of a $10 \mathrm{~nm}$ diameter precipitate. The images of He precipitates were simulated for a collection angle of 9 mrad and the rest of the images were simulated for 34 mrad. (a) line scans of the transmitted intensity taken along the centres of the precipitates starting from left to the right-hand side for the images shown in rows R1-R5.

When comparing He and Xe precipitates of a given density, a clear difference in the contrast of the two is evident. For any given density, Xe precipitates are significantly darker. This is a result of strong Z-dependence of elastic scattering cross sections. The elastic scattering cross-sections as a function of scattering angle for $300 \mathrm{keV}$ electrons are shown in Fig. 3. For a given density, high-Z elements cause a stronger electron scattering to large angles than the low-Z elements. The total elastic scattering cross-section of Xe is about 500 times the total elastic scattering cross-section of He. Due to this very strong large angle scattering, Xe precipitates appeared darker on the SON68 glass background — which is mainly contributed by low-Z elements such as $\mathrm{O}, \mathrm{Si}, \mathrm{B}$ and $\mathrm{Na}$ of the SON68 glass ( $~ 90 \%$ atomic \% combined and about 60 atomic $\%$ oxygen alone). Furthermore, on decreasing the density of the precipitates, the scattering intensity also decreases due to

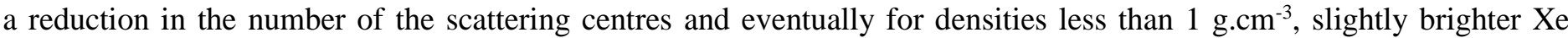
precipitates appear on the SON68 glass background. The transformation of Xe precipitates into bright voids captured using in-situ videos is shown in the supplementary video SV02-a,b. Most of the time, this transformation is so fast that a gradual contrast change is not evident. More detailed studies into this transformation - which are beyond the scope of this article - indicated that the transformation in certain cases took less than 0.2 seconds. This corresponds to gas release rates of at least $4 \times 10^{-17}$ g.s. ${ }^{-1}$ for a $20 \mathrm{~nm}$ diameter precipitate $\left(\sim 1.8 \times 10^{5}\right.$ atoms. $\left.\mathrm{s}^{-1}\right)$. 
For the case of $\mathrm{Kr}$, the simulations predicted very faint bright and dark features in the density range of our interest ( 1 to 2.3 g. $\mathrm{cm}^{-3}$ ). In fact, the brightness of the precipitates is not far from the background level (see the intensity profiles in Fig 2 (a)). This again is in excellent agreement with the experimental TEM micrographs of $\mathrm{Kr}$ precipitates shown on the righthand side in Fig 2 (a cropped region from Fig.1). Clearly distinct dark $\mathrm{Kr}$ precipitates appeared only for densities greater than $2.5 \mathrm{~g} . \mathrm{cm}^{-3}$. Such dense precipitates would require pressures $>500 \mathrm{MPa}$ corresponding to precipitates radii $<2 \mathrm{~nm}$ (from Fig. S1 in the SI). Kr precipitates with densities close to $2 \mathrm{~g} . \mathrm{cm}^{-3}$ were invisible and specimen thickness again had a small but noticeable impact on the visibility of the precipitates with densities close to $1.5 \mathrm{~g} . \mathrm{cm}^{-3}$.

For $\mathrm{Ne}$, the precipitates in the entire density range of our interest $\left(0.2\right.$ to $\left.0.8 \mathrm{~g} . \mathrm{cm}^{-3}\right)$ appeared as bright features. Ne precipitates with densities in the range of $3.5 \leq \rho \leq 5 \mathrm{~g} \mathrm{~cm}^{-3}$ were invisible. Densities greater than $5 \mathrm{~g} . \mathrm{cm}^{-3}$ were needed to observe dark appearing Ne precipitates. Such densities would require precipitate pressures in excess of $100 \mathrm{GPa}$, which is unrealistically high under implantation conditions. Therefore, Ne precipitates in glasses will always appear as bright features. However, the transformation of Ne precipitates with density $>2 \mathrm{~g} \mathrm{~cm}^{-3}$ into a void should give a visible contrast change. Density $>2 \mathrm{~g} . \mathrm{cm}^{-3}$ requires pressures $>1.5 \mathrm{GPa}$. This corresponds to Ne precipitate radii less than $1 \mathrm{~nm}$. Under normal implantation and TEM imaging conditions, the transformation of such small features is very difficult to see due to issues related to specimen roughness and its continuous modification during ion implantation. The predictions of the simulations are again in good agreement with the experimental TEM micrographs.

For the case of Ar, the precipitates in the entire density range of our interest $\left(0.5\right.$ to $\left.1.5 \mathrm{~g} . \mathrm{cm}^{-3}\right)$ appeared as bright features and this is consistent with the experimental TEM micrographs as shown on the right-hand side in Fig 2 (cropped region from Fig. 1). Densities $>4$ g. $\mathrm{cm}^{-3}$ were required to see dark appearing precipitates. This requires pressures $>50 \mathrm{GPa}$, which are impossible to achieve in the materials of interest. Thus, like $\mathrm{He}$ and $\mathrm{Ne}$, no dark precipitates can be seen during $\mathrm{Ar}$ implantation either. However, Ar precipitates with densities greater than $1 \mathrm{~g} . \mathrm{cm}^{-3}$ should result in a visible contrast change

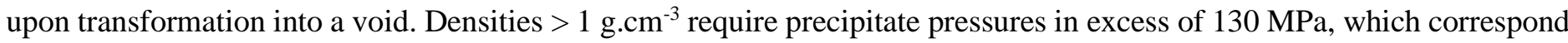
to precipitate radii $<3 \mathrm{~nm}$. Technically speaking, one should be able to see such a precipitate to void transformation in the case of Ar. When looking at the experimental TEM micrographs of Ar precipitates in Fig 1 (R3), a few spots slightly brighter than the rest can be seen (as indicated by the arrows), suggesting that the bright spots may be the voids and the rest of the features may be the Ar precipitates. However, the intensity differences are small and at the moment, we assume that it is not possible to differentiate the voids and precipitates in the case of Ar.

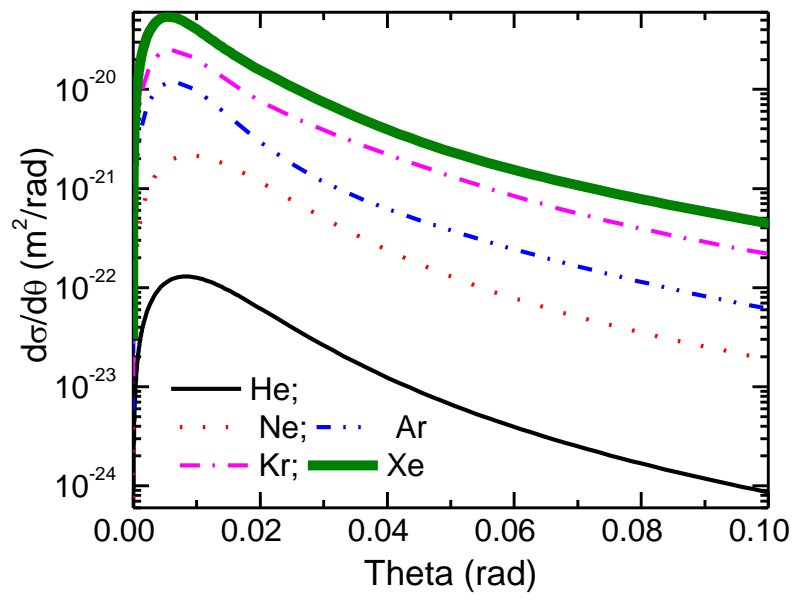

Figure-3: Elastic scattering cross-section of $300 \mathrm{keV}$ electrons for various noble gases as a function of the scattering angle. Note that for a given scattering angle, the He scattering cross-section is about two orders of magnitude smaller than the Xe scattering cross-section.

\subsubsection{Effect of precipitate and void size on their visibility}

We will now show the effect of the size of the precipitates and voids with equilibrium densities on their visibility and highlight a few complexities in differentiating the various features. The simulated BF-TEM images are shown in Fig. 4 for the case of $\mathrm{Xe}, \mathrm{Kr}$ and voids with diameters ranging from 5 to $50 \mathrm{~nm}$ in a $100 \mathrm{~nm}$ thick SON68 specimen. The diameters are written on the top side of Fig. 4 (a) and the densities of precipitates are written underneath them (equilibrium density values for the sizes under consideration). The transmitted intensity profiles are shown in Fig. 4 (b). Voids and Xe precipitates of all the sizes appeared bright and dark respectively on the SON68 background in the entire size range. Kr precipitates of 50 to $30 \mathrm{~nm}$ diameter appeared distinctly bright and the $20 \mathrm{~nm}$ precipitate was very faintly visible. Smaller 
precipitates were invisible. This is consistent with the TEM micrographs of $\mathrm{Kr}$ precipitates shown in Fig 1 (R2), a blownup region of which is shown in Fig 4 (c). Some features and their sizes in the under and at-focus images are indicated on the images in Fig 4 (c). The large voids are bright and the precipitates of similar size are faintly visible in the at-focus images as highlighted by the arrows. The intensity profiles of the voids and $\mathrm{Kr}$ precipitates in Fig 4 (b) show a noticeable intensity difference between the precipitates and the voids of similar sizes. However, the majority of small precipitates visible in the under-focused image are completely invisible in the at-focus image in Fig 4 (c). These results are in excellent agreement with the predictions of the simulations (Fig 4 (a)) showing that $\mathrm{Kr}$ precipitates of less than $20 \mathrm{~nm}$ diameter do not have enough contrast in the at-focus images. Despite a much higher noise level in the experimental images (the std dev of the background signal is about $2 \%$ and $0.3 \%$ in the experimental and simulated images), the general trends of the features seen experimentally and in the simulations are in excellent agreement with each other.

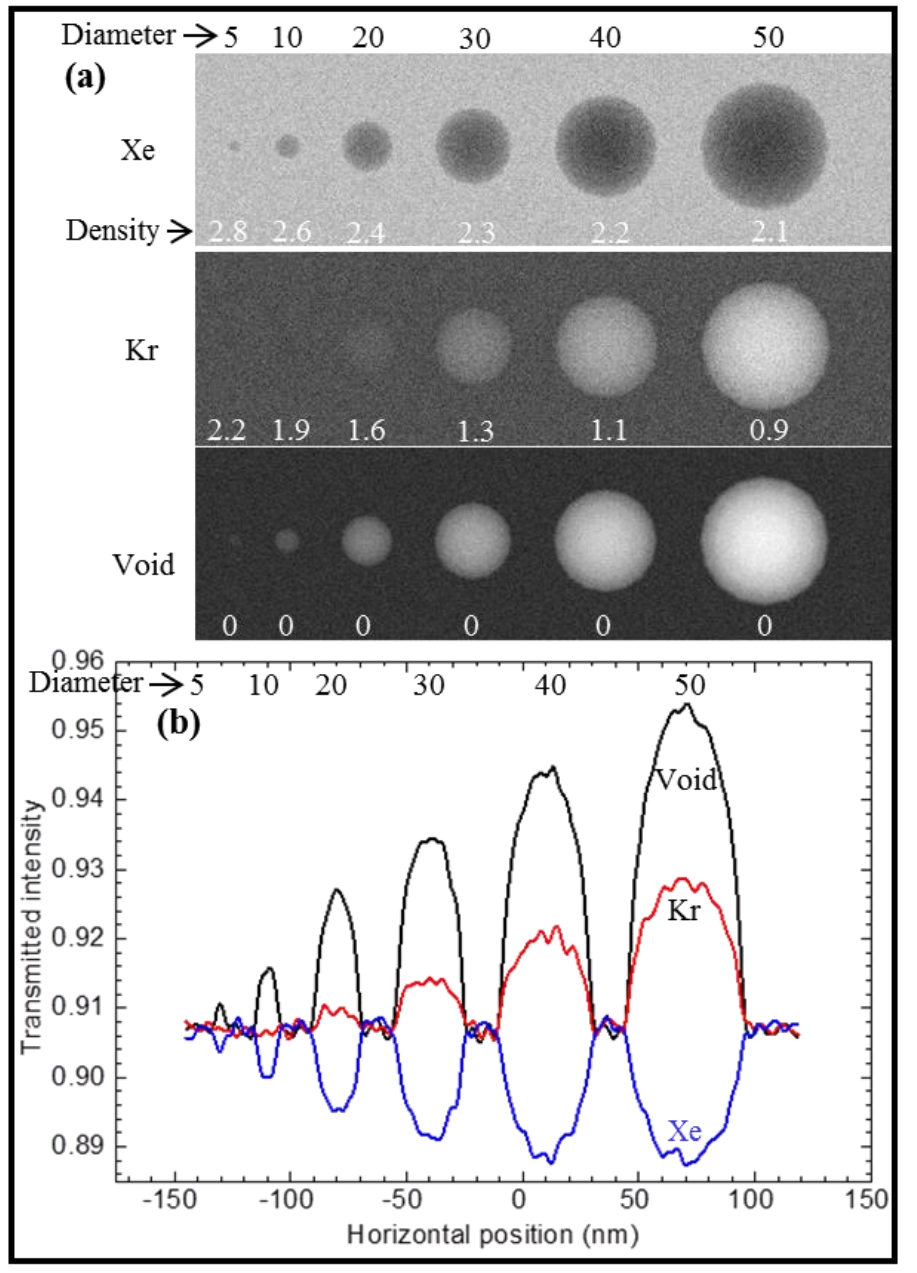

(c) Experimental TEM image of $\mathrm{Kr}$ precipitates

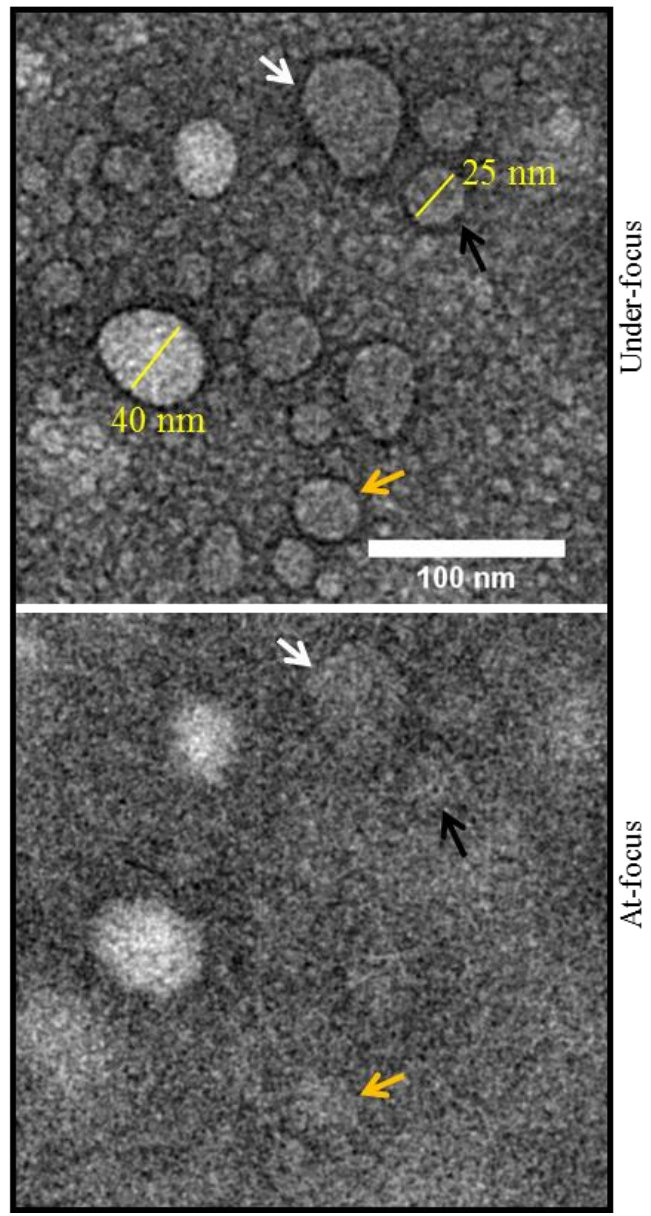

Figure-4: Simulations and experiments showing the effect of void and precipitate size on their visibility. (a), simulated BFTEM micrographs of voids, Xe and $\mathrm{Kr}$ precipitates of 5 to $50 \mathrm{~nm}$ in diameter. The densities are equilibrium precipitate densities, (b) transmitted intensity line scans taken across the voids and precipitates, (c) experimental BF-TEM image of Kr precipitates in the SON68 glass shown in under-focus (top) and at-focus (bottom) imaging conditions. Some precipitates in the two images are indicated by the arrows of the same colour.

The intensity scans shown in Fig. 4 (b) highlight a very complex situation where one might end up with misidentification of the precipitates and voids in certain cases. For instance, $\mathrm{Kr}$ precipitates of $50 \mathrm{~nm}$ diameter and voids of $20 \mathrm{~nm}$ diameter result in "almost" the same transmitted intensity. To explore this complex situation further, it is now important to provide a more quantitative sense to the term "visibility" and define where the limit of the term "almost" lies for a human eye. The appropriate term for this is referred as "contrast sensitivity", which is widely studied in the field of psychophysics and vision science. It is well established that the threshold contrast is close to $1 \%$ [37]. The contrast itself can be defined by different formulations and Weber's contrast; which is the ratio of the "intensity difference between the background ( $\left.\mathrm{I}_{\mathrm{bck}}\right)$ and the feature of interest $\left(\mathrm{I}_{\mathrm{f}}\right)$ " and the "background intensity" is used in this work. The values of Weber contrast for the simulated TEM micrographs of Fig 4 (a) are shown in Table-III. The Kr precipitate and void pairs that are indistinguishable are highlighted in the same colour $((20,5) ;(30,10)$ and $(40,20) \mathrm{nm}$ pairs). However, for a given feature size, a clear distinction between the voids and $\mathrm{Kr}$ precipitates can be made. Thus, when looking at the experimental TEM micrographs, 
one must be mindful of this issue. By properly looking at the precipitates and voids across all the sizes and comparing the contrasts of similar sizes first, a correct judgement can be made in these ambiguous situations. The Weber contrasts for all the noble gas precipitates and voids for $100 \mathrm{~nm}$ thick specimen in Fig. 2 are shown in Fig. S7 in the SI. The slopes (which reflect a change in the Weber contrast per unit change in the density) for $\mathrm{He}, \mathrm{Ne}, \mathrm{Ar}, \mathrm{Kr}$ and $\mathrm{Xe}$ are 0.04, 0.2, 0.4, 0.6, 0.7 percent per unit density change. This shows that a significant density change is required for light noble gases as compared to heavy noble gases for a visible change in the contrast. By looking at Fig. S1 (i, l) in the SI; which shows the variation of density as a function of the precipitate diameter, it is evident that very small size fluctuations are needed for small precipitates $(<2 \mathrm{~nm})$ to induce a visible contrast change due to a steep slope of the density curve whereas for large precipitates, very large size changes are needed to induce a visible contrast change. Thus, the contrasts of small precipitates or highly pressurized precipitates of heavy noble gases are very susceptible to slight fluctuations in the precipitate sizes and this susceptibility decreases with $\mathrm{Z}$ of the noble gas atom.

Table-IV: Normalized transmitted intensity and Weber contrast of the voids and precipitates of various sizes shown in Fig. $4\left(\right.$ a). $I_{b c k}, I_{V o i d}, I_{X e}, I_{K r}$ are the normalized transmitted intensities for background, void, Xe and Kr precipitates respectively.

\begin{tabular}{|c|c|c|c|c|c|c|}
\hline \multicolumn{7}{|c|}{$\mathbf{I}_{\text {bck }}=\mathbf{0 . 9 0 7} \pm \mathbf{0 . 0 0 2 8}$, Specimen thickness = 100 nm } \\
\hline $\begin{array}{c}\text { Diameter } \\
(\mathbf{n m})\end{array}$ & $\mathbf{I K r}$ & $\begin{array}{c}\text { Weber } \\
\text { contrast } \\
(\boldsymbol{\%})\end{array}$ & $\mathbf{I x e}$ & $\begin{array}{c}\text { Weber } \\
\text { contrast } \\
\mathbf{\%})\end{array}$ & IVoid & $\begin{array}{c}\text { Weber } \\
\text { contrast } \\
\mathbf{\%})\end{array}$ \\
\hline $\mathbf{5}$ & 0.907 & 0 & 0.9035 & -0.4 & 0.91077 & 0.4 \\
\hline $\mathbf{1 0}$ & 0.907 & 0 & 0.8998 & -0.8 & 0.9159 & 1 \\
\hline $\mathbf{2 0}$ & 0.91 & 0.3 & 0.8947 & -1.4 & 0.9269 & 2.2 \\
\hline $\mathbf{3 0}$ & 0.914 & $\mathbf{0 . 8}$ & 0.89085 & -1.8 & 0.9344 & 3 \\
\hline $\mathbf{4 0}$ & 0.921 & $\mathbf{1 . 5}$ & 0.8873 & -2.2 & 0.94436 & 4.1 \\
\hline $\mathbf{5 0}$ & 0.9285 & 2.4 & 0.88677 & -2.2 & 0.9538 & 5.2 \\
\hline
\end{tabular}

From the results and discussions presented so far, a few important points that are worth highlighting and remembering with regards to at-focus BF-TEM images of the noble gas precipitates in glasses are listed below:

I. Precipitates of heavy noble gases like $\mathrm{Xe}$ and $\mathrm{Kr}$ can be easily identified and differentiated from voids by using at-focus TEM images.

II. Xe precipitates over a wide range of sizes are visible as dark features in the over and at-focus images. $\mathrm{Kr}$ precipitates appear relatively lighter but clearly distinguishable from the bright voids.

III. Although large Kr precipitates and small voids can have similar contrasts - which can create an ambiguity in their differentiation - comparing features of similar sizes first can help to differentiate a precipitate from a void in its own size group. When doing similar comparison across all the sizes, a clear distinction between the precipitates and voids can be made.

IV. The precipitates of Ar and voids are difficult, if not impossible, to differentiate. But more work is needed on the subject.

V. The precipitates of $\mathrm{He}$ and $\mathrm{Ne}$ cannot be distinguished from voids. This is primarily due to the very low elastic scattering cross-sections of low-Z atoms. In addition, the low densities of light-noble-gases make it impossible to distinguish their precipitates from the voids even up to pressures unlikely to be encountered during implantation of glasses or any other minerals and metals with surface tensions even an order of magnitude higher.

VI. A significant density change is needed for light noble gas precipitates to induce a visible contrast change. Assuming $1 \%$ contrast change is the visibility threshold, density changes of about 1.4, 1.7, 2.5, 5 and 25 units are needed for $\mathrm{Xe}, \mathrm{Kr}, \mathrm{Ar}, \mathrm{Ne}$ and $\mathrm{He}$ respectively (density change per unit change in the contrast). This shows that isochronal annealing studies can be easily performed on $\mathrm{Xe}$ and $\mathrm{Kr}$ precipitates to study the diffusionmediated noble gas loss and contrast changes as a result.

VII. $\quad \mathrm{Z}$ of the scattering atom plays a more dominant role than the density of the precipitates. This is because the density of various noble gas precipitates varies within an order of magnitude only whereas the scattering crosssections vary by up to two orders of magnitude. 


\subsubsection{Differentiating the precipitates and voids of light noble gases}

For now, we are left with the question of whether it is at all possible to differentiate the voids and precipitates of light noble gases like $\mathrm{He}$ and $\mathrm{Ne}$ using BF-TEM images. Leaving aside what can be directly observed or inferred from the BF-TEM micrographs, continuous in-situ monitoring of the inclusions provides indispensable clues to differentiate the precipitates and voids even for light noble gases like $\mathrm{He}$ and $\mathrm{Ne}$. To understand how the precipitates and voids behaved under continuous ion irradiation, short in-situ videos were recorded. These videos are shown in the SI (videos SV01 for Xe in $\mathrm{SiO}_{2}$, SV02a,b for Xe in SON68, SV03 for Ne in SON68, SV04 for He in SON68). The videos showed that the precipitates grew and, after remaining stable under ion irradiation for a certain time period, quickly transformed into voids (the initial void had the same size as the parent precipitate). Once a void was formed, it started to shrink in size and eventually closed as the ion irradiation continued. The regions where the voids disappeared appeared with a slightly brighter background in the TEM micrographs as can be seen in the videos and indicated in the TEM micrograph in Fig S8 in the SI. Some of the data on how fast the voids closed during ion irradiation is shown in Fig. S9 in the SI for $35 \mathrm{keV} \mathrm{Kr}$ irradiation of the SON68 glass. The total number of ion impacts needed to close a void was proportional to the initial size of the void. A void of $20 \mathrm{~nm}$ radius required about $10^{4}$ ion impacts in total to completely close. However, the number of ion impacts needed per unit area of the void to close was found to be constant for the voids of various sizes ( $~ 9 \pm 2$ ion impacts per unit area). Similar studies for the case of $45 \mathrm{keV} \mathrm{Xe}$ ion irradiation (not shown) yielded $5 \pm 1$ ion impacts per unit area. Currently, there are no systematic studies on the impact of ion energy and ion type on the number of ion impacts needed for void closure. But, one would expect fewer impacts needed for heavy ions than for the light ions due to higher ballistic damage associated with heavy ions.

The important observation to highlight here is that the precipitates grew and remained stable after attaining a certain size whereas the voids were unstable to ion irradiation and continuously shrank and eventually closed due to ion irradiation. Therefore, even though it is not possible to distinguish the light-noble-gas precipitates and voids in an isolated BF-TEM micrograph, a history of their evolution recorded using in-situ experiments can be used to differentiate the precipitates and voids based on their behaviour. These observations also underline the pitfalls in clearly differentiating the bubble/precipitate re-solution from void closure. If proper care is not taken, the void closure can be easily mistaken for bubble/precipitate resolution. More details about the fundamental processes leading to void closure and precipitate growth are given elsewhere [38]. Based on the MD simulation of effects of ballistic collisions on the voids in amorphous silicon, it is known that the void closure results due to a combination of viscous flow, and convective flow towards the void due to momentum transfer during ballistic collisions [39-41]. The high-pressure precipitates, on the other hand, prevent the convective flow towards the precipitates during the ballistic phase and provide stability against the shrinkage.

\section{Conclusion}

This study explored the formation of noble gas precipitates and voids in glasses using TEM with in-situ ion implantation. The main focus was to show how the atomic number, specimen thickness, size and, density of the precipitates affected their visibility. Simulations of the TEM images of precipitates and voids using Monte-Carlo methods and appropriate equations of state of the noble gases were also performed. The study showed that $\mathrm{Xe}$ and $\mathrm{Kr}$ precipitates due to their high density and high elastic scattering cross-sections can be easily differentiated from voids in the TEM micrographs. Although, there are certain void and $\mathrm{Kr}$ precipitate size combinations which are difficult to distinguish from each other, this type of ambiguity can be resolved by comparing and contrasting the precipitates and voids in various size groups and then identifying the individual features based on this grouping. Helium, $\mathrm{Ne}$ and Ar precipitates on the other hand due to their low density and low scattering cross-sections cannot be differentiated from voids in the TEM micrographs. Although it is not possible to directly distinguish the precipitates and voids of $\mathrm{He}, \mathrm{Ne}$ and $\mathrm{Ar}$ from the TEM images, it is possible to make such a distinction using continuous in-situ monitoring and exploiting the intrinsic behaviour of the precipitates and voids under ion irradiation. From our studies, we conclude that the precipitates after a certain degree of growth transform into voids. The voids, unlike precipitates, are unstable under ion irradiation and shrink in size and eventually close upon continuous ion irradiation. This inherent behaviour of the precipitates and voids allows one to differentiate between them even in the case of light noble gases. The results also highlight that the unstable nature of the voids can easily be mistaken as re-solution of the precipitates. Such differentiation is essential in developing accurate models for the evolution of precipitates/bubbles and voids in a number of nuclear materials, especially, nuclear fuel pellets and waste matrices. It is also important to highlight that amongst all the noble gases, studies on the evolution and dynamics of $\mathrm{Xe}$ and $\mathrm{Kr}$ precipitates in amorphous materials during ion implantation as well as during annealing offer the best model system to understand in detail the processes such as growth, coalescence and re-solution of the precipitates due to the possibility of unambiguous differentiation of the precipitates and voids. 


\section{Acknowledgement}

The authors acknowledge the funding grants EP/E017266/1, EP/M011135/1 and EP/M028283/1 from Engineering and Physical Sciences Research Council of the United Kingdom.

\section{Author contribution}

A. H. Mir planned and performed the in-situ TEM experiments and MC simulations. A.H Mir, J.A. Hinks and S.E. Donnelly performed the data analysis and interpreted the results. All the authors contributed to writing and reviewing the manuscript.

\section{Data Availability statement}

The raw data (images and videos) can be made available as individual files on request but can not be uploadedat this time due to file sizes in the range of tens of giga bytes. 


\section{References:}

[1] E. Oliviero, M.O. Ruault, B. Décamps, F. Fotuna, E. Ntsoenzok, O. Kaïtasov, S. Collin, Synthesis of mesoporous amorphous silica by $\mathrm{Kr}$ and $\mathrm{Xe}$ ion implantation: Transmission electron microscopy study of induced nanostructures, Microporous Mesoporous Mater. 132 (2010) 163-173. doi:10.1016/j.micromeso.2010.02.015.

[2] Y. Zhao, D. Liu, J. Chen, L. Zhu, A. Belianinov, O.S. Ovchinnikova, R.R. Unocic, M.J. Burch, S. Kim, H. Hao, D.S. Pickard, B. Li, J.T.L. Thong, Engineering the thermal conductivity along an individual silicon nanowire by selective helium ion irradiation, Nat. Commun. 8 (2017) 1-8. doi:10.1038/ncomms15919.

[3] B. Lorenzi, S. Frabboni, G.C. Gazzadi, R. Tonini, G. Ottaviani, D. Narducci, Nanovoid Formation and Dynamics in He+ Implanted Nanocrystalline Silicon, J. Electron. Mater. 43 (2014) 3852-3856. doi:10.1007/s11664-0143249-4.

[4] V. Vishnyakov, S.E. Donnelly, G. Carter, R. Birtcher, L. Haworth, Helium Bubble Growth in Silicon: Ripening or Bubble Motion and Coalescence?, Solid State Phenom. 82-84 (2002) 267-272. doi:10.4028/www.scientific.net/SSP.82-84.267.

[5] K.J. Stevens, P.B. Johnson, Defocussed TEM contrast from small helium bubbles in copper, J. Nucl. Mater. 246 (1997) 17-29. http://www.sciencedirect.com/science/article/B6TXN-3SP5M103J/2/9a8bf6eca43b3ced877f71f0bd175129.

[6] S.E. Donnelly, R.C. Birtcher, C. Templier, The effect of dense and dilute collision cascades on helium bubbles in metals, Nucl. Inst. Methods Phys. Res. B. 106 (1995) 583-588. doi:10.1016/0168-583X(95)00774-1.

[7] S.E. Donnelly, R.C.Bircher, C.Templier, V.Vishnyakov, Response of helium bubbles in gold to displacementcascade damage, 52 (1995).

[8] P.B. Johnson, D.J. Mazey, Gas-bubble superlattice formation in bcc metals, J. Nucl. Mater. 218 (1995) 273-288. doi:10.1016/0022-3115(94)00674-1.

[9] R.C.Birtcher, S.E.Donnelly, T. Templier, Evolution of helium bubbles in aluminium during heavy-ion irradiation, Phys. Rev. B. 50 (1994) 764.

[10] R. Evron, Y. Cohen, O. Regev, Y. Eyal, H. Matzke, K. Tinschert, Ion implantation induced microstructural damage in a nuclear waste glass, Nucl. Soc. Isr. 18 (1994) VIII-2-VIII-7.

[11] P.B. Johnson, K.J. Stevens, R.W. Thomson, Image processing techniques in the study of ordered bubble arrays: bubble facetting in vanadium, Nucl. Inst. Methods Phys. Res. B. 62 (1991) 218-227. doi:10.1016/0168583X(91)95902-P.

[12] D.R. Olander, D. Wongsawaeng, Re-solution of fission gas - A review: Part I. Intragranular bubbles, J. Nucl. Mater. 354 (2006) 94-109. doi:10.1016/j.jnucmat.2006.03.010.

[13] N. Marochov, P.J. Goodhew, A comparison of the growth of helium and neon bubbles in nickel, J. Nucl. Mater. 158 (1988) 81-86. doi:10.1016/0022-3115(88)90157-2.

[14] J. Noordhuis, J. De Hosson, The Influence of Noble Gas Bubbles On Mechanical Properties of Steel, Scr. Metall. Mater. 25 (1991) 539-542. http://www.sciencedirect.com/science/article/pii/0956716X9190087H.

[15] A. Luukkainen, J. Keinonen, M.Erola, Density and size of neon bubbles in molyddenum. tantalum, and tantalum oxide, Phys. Rev. B. 32 (1985) 4814-4817.

[16] A. Vom Felde, J. Fink, T. M̈ller-Heinzerling, J. Pfïger, B. Scheerer, G. Linker, D. Kaletta, Pressure of neon, argon, and xenon bubbles in aluminum, Phys. Rev. Lett. 53 (1984) 922-925. doi:10.1103/PhysRevLett.53.922.

[17] C.J. Rossouw, S.E. Donnelly, Superheating of small solid-argon bubbles in aluminum, Phys. Rev. Lett. 55 (1985) 2960-2963. doi:10.1103/PhysRevLett.55.2960.

[18] A.S. Lis, R.C.Birtcher, Precipitation of $\mathrm{Ar}, \mathrm{Kr}$ and $\mathrm{Xe}$ in Ni at Room Temperature, in: MRS Symp. Charact. Mater. Using Ion Beams, 1988. doi:https://doi.org/10.1557/PROC-128-303.

[19] L. Pagano, A.T. Motta, R.C. Birtcher, The formation of bubbles in $\mathrm{Zr}$ alloys under $\mathrm{Kr}$ ion irradiation, J. Nucl. Mater. 244 (1997) 295-304. doi:10.1016/S0022-3115(96)00757-X.

[20] R.C. Birtcher, A.S. Liu, Temperature dependence of Kr precipitation in Ni, J. Nucl. Mater. 165 (1989) 101-109. doi:10.1016/0022-3115(89)90238-9.

[21] J. Rest, R.C. Birtcher, Precipitation kinetics of rare gases implanted into metals, J. Nucl. Mater. (1989) 312-325.

[22] J.H. Evans, D.J. Mazey, Evidence for solid krypton bubbles in copper, nickel and gold at 293K, J. Phys. F Met. Phys. 15 (1985). doi:10.1088/0305-4608/15/1/001.

[23] L. He, X.M. Bai, J. Pakarinen, B.J. Jaques, J. Gan, A.T. Nelson, A. El-Azab, T.R. Allen, Bubble evolution in Krirradiated UO2 during annealing, (2017). doi:10.1016/j.jnucmat.2017.09.036.

[24] N. Ishikawa, M. Awaji, K. Furuya, R.C. Birtcher, C.W. Allen, HRTEM analysis of solid precipitates in Xeimplanted aluminum, Nucl. Instruments Methods Phys. Res. Sect. B Beam Interact. with Mater. Atoms. 127-128 (1997) 123-126. doi:10.1016/S0168-583X(96)00864-6.

[25] L. Shartsis, A.W. Smock, Surface Tensions of Some Optical Glasses, J. Am. Ceram. Soc. 30 (1947) 130-136. doi:10.1111/j.1151-2916.1947.tb18881.x.

[26] G. Greaves, A.H. Mir, R.W. Harrison, M.A. Tunes, S.E. Donnelly, J.A. Hinks, New Microscope and Ion 
Accelerators for Materials Investigations (MIAMI-2) system at the University of Huddersfield, Nucl. Instruments Methods Phys. Res. Sect. A Accel. Spectrometers, Detect. Assoc. Equip. 931 (2019) 37-43. doi:10.1016/j.nima.2019.03.074.

[27] J.F. Ziegler, M.D. Ziegler, J.P. Biersack, SRIM - The stopping and range of ions in matter (2010), Nucl. Inst. Methods Phys. Res. B. 268 (2010) 1818-1823. doi:10.1016/j.nimb.2010.02.091.

[28] P. Hovington, D. Drouin, R. Gauvin, CASINO: A new monte carlo code in C language for electron beam interaction -part I: Description of the program, Scanning. 19 (2006) 1-14. doi:10.1002/sca.4950190101.

[29] H. Demers, N. Poirier-Demers, A.R. Couture, D. Joly, M. Guilmain, N. De Jonge, D. Drouin, Three-dimensional electron microscopy simulation with the CASINO Monte Carlo software, Scanning. 33 (2011) 135-146. doi:10.1002/sca.20262.

[30] H. Demers, N. Poirier-Demers, D. Drouin, N. De Jonge, Simulating STEM Imaging of Nanoparticles in Micrometers-Thick Substrates, Microsc.Microanal. 16 (2010) 795-804. doi:10.1017/S1431927610094080.

[31] D. Drouin, A.E. Couture, D. Joly, X. Tastet, V. Aimez, R. Gauvin, CASINO V2.42 - A Fast and Easy-to-use Modeling Tool for Scanning Electron Microscopy and Microanalysis Users, Scanning. 29 (2007) 92-101.

[32] F. Salvat, A. Jablonski, C.J. Powell, Elsepa - Dirac partial-wave calculation of elastic scattering of electrons and positrons by atoms, positive ions and molecules, Comput. Phys. Commun. 165 (2005) 157-190. doi:10.1016/j.cpc.2004.09.006.

[33] C.W. Lee, Y. Ikematsu, D. Shindo, Measurement of mean free paths for inelastic electron scattering of Si and $\mathrm{SiO} 2$, J. Electron Microsc. (Tokyo). 51 (2002) 143-148. doi:10.1093/jmicro/51.3.143.

[34] R.F. Egerton, Electron energy loss spectroscopy in the microscope (Page No. 293), 2nd Ed., Plenum publishing coorporation, 1989.

[35] D.F. Swinehart, The Beer-Lambert Law, J. Chem. Educ. 39 (1962) 333. doi:10.1021/ed039p333.

[36] A.H. Mir, J.A. Hinks, J.-M. Delaye, S. Peuget, S.E. Donnelly, Xenon solubility and formation of supercritical xenon precipitates in glasses under non-equilibrium conditions, Sci. Rep. 8 (2018) 15320. doi:10.1038/s41598018-33556-y.

[37] D.G. Pelli, P. Bex, Measuring contrast sensitivity, Vision Res. 90 (2013) 10-14. doi:10.1016/j.visres.2013.04.015.

[38] A.H. Mir, J. Hinks, S.E.Donnelly, Ballistic-damage-induced size changes in equilibrium and under-pressurized Xe precipitates in amorphous silica, J. Nucl. Mater. 519 (2019) 229-238.

[39] M.A. Okunlewski, Y. Ashkenazy, B.J. Heuser, R.S. Averback, Molecular dynamics simulations of void and helium bubble stability in amorphous silicon during heavy-ion bombardment, J. Appl. Phys. 96 (2004) 4181-4188. doi:10.1063/1.1791759.

[40] X.F. Zhu, J.S. Williams, M.J. Conway, M.C. Ridgway, F. Fortuna, M.O. Ruault, H. Bernas, Direct observation of irradiation-induced nanocavity shrinkage in Si, Appl. Phys. Lett. 79 (2001) 3416-3418. doi:10.1063/1.1413497.

[41] M.O. Ruault, F. Fortuna, H. Bernas, M.C. Ridgway, J.S. Williams, How nanocavities in amorphous Si shrink under ion beam irradiation: An in situ study, Appl. Phys. Lett. 81 (2002) 2617-2619. doi:10.1063/1.1509854. 